\title{
Gravitational wave background from black holes of the Springel \& Hernquist star formation epoch
}

\author{
José C. N. de Araujo, ${ }^{1 \star}$ Oswaldo D. Miranda ${ }^{2 \star}$ and Odylio D. Aguiar ${ }^{1 \star}$ \\ ${ }^{1}$ Instituto Nacional de Pesquisas Espaciais, Divisão de Astrofísica, Av. dos Astronautas 1758, SJ dos Campos, 12227-010 SP, Brazil \\ ${ }^{2}$ Instituto Tecnológico de Aeronáutica, Departamento de Física, Pr. Mar. Eduardo Gomes 50, SJ dos Campos, $12228-900$ SP, Brazil
}

Accepted 2003 November 20. Received 2003 November 17; in original form 2003 March 26

\begin{abstract}
We study the generation of a stochastic gravitational wave (GW) background produced from a population of core-collapse supernovae, which form black holes in scenarios of structure formation. In particular, we take into account in the present paper the history of star formation derived by Springel \& Hernquist, who employed hydrodynamic simulations of structure formation in a $\Lambda$ cold dark matter $(\Lambda \mathrm{CDM})$ cosmology. In addition, and contrary to previous studies, we consider the effect of taking into account different values of the $\alpha$ parameter, the fraction of the progenitor mass which forms the black hole. Different values of $\alpha$ imply different frequency bands for the GWs produced. Because the sensitivity of the GW detectors depends on the frequency of the GWs, it is worth studying the influence of $\alpha$ on the detection of the background considered here. We then study the detectability of the GW background produced in scenarios such as those studied here and discuss what astrophysical information could be obtained from a positive - or even negative - detection of such a putative background. In particular, we show that the formation of a pre-galactic population of black holes could have generated a stochastic GW background that should be detected in the future by correlating signals of a pair of 'advanced' LIGO observatories (LIGO III) at a signal-to-noise ratio greater than unity, if $\alpha>0.5$.
\end{abstract}

Key words: black hole physics - gravitation - gravitational waves - cosmology: theory.

\section{INTRODUCTION}

Because of the fact that gravitational waves (GWs) are produced by a large variety of astrophysical sources and cosmological phenomena, it is quite probable that the Universe is pervaded by a background of such waves. A variety of binary stars (ordinary, compact or combinations of them), Population III stars, phase transitions in the early Universe and cosmic strings are examples of sources that could produce such putative GW background (Thorne 1987).

As GWs possess a very weak interaction with matter, passing through it without being disturbed, once detected they can provide information on the physical conditions from the era in which they were produced. In principle, it will be possible to get information from the epoch when the galaxies and stars started to form and evolve.

We consider in the present paper the putative background of GWs generated during the formation of black holes in a scenario of structure formation recently studied by Springel \& Hernquist (2003).

Recall that, when the high-mass stars died as supernovae, they left stellar black holes as remnants. The formation of these stellar black holes can, in principle, produce a GW background detectable by

\footnotetext{
^E-mail: jcarlos@das.inpe.br(JCNA); oswaldo@fis.ita.br(ODM); odylio@ das.inpe.br (ODA)
}

GW observatories. It is worth mentioning that a significant amount of GWs can also be produced during the formation of neutron stars. However, because this depends on the equation of state for the neutron star, which is not well defined, we consider here only the contribution of the black holes. Another possibility would be the generation of GWs through the so-called r-mode instability (Anderson 1998), which should be important for young, hot and rapidly rotating neutron stars, but we leave this issue for another study to appear elsewhere.

Springel \& Hernquist (2003) obtain the history of star formation from hydrodynamic simulations of structure formation in $\Lambda$ cold dark matter $(\Lambda \mathrm{CDM})$ cosmology. They study the history of cosmic star formation from the 'dark ages', at redshift $z \sim 20$, to the present. Besides gravity and ordinary hydrodynamics, they take into account radiative heating and cooling of gas, supernova feedbacks and galactic winds. Their paper improves previous studies, which consider either semi-analytical models or numerical simulations.

It is worth mentioning that the story of star formation they obtain is consistent with observations. It is important to bear in mind, however, that nowadays observations give information from at most a redshift around $z \sim 5$. In the future, however, with the Next Generation Space Telescope (NGST), it will be possible to trace the cosmic star formation rate out to $z \gtrsim 20$ (see, for example, Mackey, Bromm \& Hernquist 2003). 
Besides the reliable history of star formation by Springel \& Hernquist (2003), we consider the role of the parameter $\alpha$, the fraction of the progenitor mass which forms the remnant black hole. Recall that a given initial mass function (IMF) refers to the distribution function of the stellar progenitor mass, and also to the masses of the remnant compact objects left as a result of the stellar evolution.

In our previous studies (de Araujo, Miranda \& Aguiar 2000, 2002a,b), we have fixed $\alpha=0.1$ while Ferrari, Matarrese \& Schneider (1999) adopted $\alpha=0.05$ and 0.2 in addition. However, these values of $\alpha$ are probably not adequate for progenitor masses which give rise to black holes.

In fact, the mass of the ejecta from a dying star depends sensitively on the stellar metallicity, with low- $Z$ stars having higher remnants masses and less ejected material relative to solar- $Z$ stars. It is expected that between $\sim 50$ and 95 per cent of the mass of the parent star as ejecta, until a point is reached where the entire star collapses into a black hole (see, for example, Venkatesan 2000), which means $\alpha \sim 1$.

In the present study, we have adopted a stellar generation with a Salpeter IMF, a history of star formation given by Springel \& Hernquist (2003), as well as different values of $\alpha$. We then discuss what conclusions would be drawn whether (or not) the stochastic background studied here is detected by the forthcoming GW observatories such as LIGO and VIRGO.

The paper is organized as follows. In Section 2, we describe how to calculate the background of GWs produced during the formation of the stellar black holes in the Springel \& Hernquist scenario; in Section 3, we present and discuss the numerical results and the detectability of this putative GW background; and finally in Section 4, we present the conclusions.

\section{GRAVITATIONAL WAVE PRODUCTION FROM THE SPRINGEL \& HERNQUIST STELLAR FORMATION EPOCH}

Let us focus on how to calculate the background of GWs produced by a population of stellar black holes in the scenario studied by Springel \& Hernquist (2003). Following de Araujo, Miranda \& Aguiar (2000; see also de Araujo, Miranda \& Aguiar 2001, 2002a,b), the dimensionless amplitude, $h_{\mathrm{BG}}$, of the stochastic $\mathrm{GW}$ background produced by gravitational collapses that lead to black holes is given by

$h_{\mathrm{BG}}^{2}=\frac{1}{v_{\mathrm{obs}}} \int h_{\mathrm{BH}}^{2} \mathrm{~d} R_{\mathrm{BH}}$,

where $\mathrm{d} R_{\mathrm{BH}}$ is the differential rate of black hole formation, $h_{\mathrm{BH}}$ is the dimensionless amplitude produced by the collapse to a black hole that generates at the present time a signal with frequency $v_{\text {obs }}$.

The dimensionless amplitude produced by the collapse of a star, or star cluster, to form a black hole is (Thorne 1987)

$$
\begin{aligned}
h_{\mathrm{BH}} & =\left(\frac{15}{2 \pi} \varepsilon_{\mathrm{GW}}\right)^{1 / 2} \frac{G}{c^{2}} \frac{M_{\mathrm{r}}}{d_{\mathrm{L}}} \\
& \simeq 7.4 \times 10^{-20} \varepsilon_{\mathrm{GW}}^{1 / 2}\left(\frac{M_{\mathrm{r}}}{\mathbf{M}_{\odot}}\right)\left(\frac{d_{\mathrm{L}}}{1 \mathrm{Mpc}}\right)^{-1},
\end{aligned}
$$

where $\varepsilon_{\mathrm{GW}}$ is the efficiency of generation of GWs, $M_{\mathrm{r}}$ is the mass of the black hole formed and $d_{\mathrm{L}}$ is the luminosity distance to the source.

It is worth mentioning that equation (2) refers to the black hole 'ringing', which has to do with the de-excitation of the black hole quasi-normal modes. Note also that $\varepsilon_{\mathrm{GW}} \propto a^{4}$ (see, for example, Stark \& Piran 1986), where $a$ is the dimensionless angular momentum. Thus, the greater the GW efficiency, the greater the dimensionless angular momentum.

The collapse of a star to a black hole produces a signal with frequency (Thorne 1987)

$$
\begin{aligned}
v_{\mathrm{obs}} & =\frac{1}{5 \pi M_{\mathrm{r}}} \frac{c^{3}}{G}(1+z)^{-1} \\
& \simeq 1.3 \times 10^{4} \mathrm{~Hz}\left(\frac{\mathrm{M}_{\odot}}{M_{\mathrm{r}}}\right)(1+z)^{-1},
\end{aligned}
$$

where the factor $(1+z)^{-1}$ takes into account the redshift effect on the emission frequency, that is, a signal emitted at frequency $v_{\mathrm{e}}$ at redshift $z$ is observed at frequency $v_{\text {obs }}=v_{\mathrm{e}}(1+z)^{-1}$.

For the differential rate of black hole formation we have

$\mathrm{d} R_{\mathrm{BH}}=\frac{\dot{\rho}_{\star}(z)}{(1+z)} \frac{\mathrm{d} V}{\mathrm{~d} z} \phi(m) \mathrm{d} m \mathrm{~d} z$

where $\dot{\rho}_{\star}(z)$ is the star formation rate (SFR) density, $\phi(m)$ is the IMF and $\mathrm{d} V$ is the comoving volume element.

From the above equations, we obtain for the dimensionless amplitude

$$
\begin{aligned}
h_{\mathrm{BG}}^{2}= & \frac{\left(7.4 \times 10^{-20} \alpha\right)^{2} \varepsilon_{\mathrm{GW}}}{v_{\mathrm{obs}}} \\
& \times\left[\int_{z_{\mathrm{cf}}}^{z_{\mathrm{ci}}} \int_{m_{\min }}^{m_{\mathrm{u}}}\left(\frac{m}{\mathrm{M}_{\odot}}\right)^{2}\left(\frac{d_{\mathrm{L}}}{1 \mathrm{Mpc}}\right)^{-2} \frac{\dot{\rho}_{\star}(z)}{(1+z)}\right. \\
& \left.\times \frac{\mathrm{d} V}{\mathrm{~d} z} \phi(m) \mathrm{d} m \mathrm{~d} z\right] .
\end{aligned}
$$

We consider that $M_{\mathrm{r}}=\alpha m$, where $m$ is the progenitor mass for the black holes, which ranges from $m_{\min }-m_{\mathrm{u}}=25-125 \mathrm{M}_{\odot}$.

Note that the above equation takes into account the contribution of different masses that collapse to form black holes at redshifts ranging from $z_{\mathrm{ci}}$ to $z_{\mathrm{cf}}$ (beginning and end of the star formation phase, respectively) that produce a signal at the same frequency $v_{\mathrm{obs}}$.

The comoving volume element is given by

$\mathrm{d} V=4 \pi\left(\frac{c}{H_{0}}\right) r_{\mathrm{z}}^{2} \mathcal{F}\left(\Omega_{\mathrm{M}}, \Omega_{\Lambda}, z\right) \mathrm{d} z$,

with

$$
\mathcal{F}\left(\Omega_{\mathrm{M}}, \Omega_{\Lambda}, z\right) \equiv \frac{1}{\sqrt{(1+z)^{2}\left(1+\Omega_{\mathrm{M}} z\right)-z(2+z) \Omega_{\Lambda}}},
$$

and the comoving distance, $r_{\mathrm{z}}$, is

$r_{\mathrm{z}}=\frac{c}{H_{0} \sqrt{\left|\Omega_{\mathrm{k}}\right|}} S\left[\sqrt{\left|\Omega_{\mathrm{k}}\right|} \int_{0}^{z} \frac{\mathrm{d} z^{\prime}}{\mathcal{F}\left(\Omega_{\mathrm{M}}, \Omega_{\Lambda}, z^{\prime}\right)}\right]$,

where

$\Omega_{\mathrm{M}}=\Omega_{\mathrm{DM}}+\Omega_{\mathrm{B}} \quad$ and $\quad 1=\Omega_{\mathrm{k}}+\Omega_{\mathrm{M}}+\Omega_{\Lambda}$

are the usual density parameters for the matter (M), i.e. dark matter (DM) plus baryonic matter (B), curvature (k) and cosmological constant $(\Lambda)$. The function $S$ is given by

$S(x)= \begin{cases}\sin x & \text { if closed, } \\ x & \text { if flat, } \\ \sinh x & \text { if open. }\end{cases}$

The comoving distance is related to the luminosity distance by

$d_{\mathrm{L}}=r_{\mathrm{z}}(1+z)$.

The set of equations presented above can be used to find the dimensionless amplitude $h_{\mathrm{BG}}$ of the GW background generated by black hole formation for given SFR density and IMF. 
Here, the Salpeter IMF is adopted, namely

$\phi(m)=A m^{-(1+x)}$,

where $A$ is the normalization constant and $x=1.35$ (our fiducial value). The normalization of the IMF is obtained through the relation

$\int_{m_{1}}^{m_{\mathrm{u}}} m \phi(m) \mathrm{d} m=1$,

where we consider $m_{1}=0.1 \mathrm{M}_{\odot}$ and $m_{\mathrm{u}}=125 \mathrm{M}_{\odot}$. It is worth noting that some authors argue (see, for example, Gilmore 2001) that there is evidence supporting the universality of the IMF, even for the first stars.

On the other hand, some authors (see, for example, Scalo 1998, among others) argue that the IMF may not be universal. In particular, the universality of the Salpeter exponent $(x=1.35)$ has been studied by recent evolutionary models for the Magellanic Clouds (de Freitas-Pacheco 1998). Some models, particularly those of the Large Magellanic Cloud, take into account constraints on the star formation history imposed by recent data on colour-magnitude diagrams of field star clouds, showing that a steeper exponent $(x=2.0)$ is necessary to resolve the excessive production of iron obtained if one takes into account the Salpeter law $(x=1.35)$. Furthermore, concerning the star formation at high redshift, the IMF could be biased toward high-mass stars, when compared to the solar neighbourhood IMF, as a result of the absence of metals (Bromm, Coppi \& Larson 1999, 2002).

For the SFR density, we consider the one derived by Springel \& Hernquist (see Springel \& Hernquist 2003 for details), namely

$\dot{\rho}_{\star}(z)=\dot{\rho}_{\mathrm{m}} \frac{\beta \exp \left[\Delta\left(z-z_{\mathrm{m}}\right)\right]}{\beta-\Delta+\Delta \exp \left[\beta\left(z-z_{\mathrm{m}}\right)\right]}$,

where $\Delta=3 / 5, \beta=14 / 15, z_{\mathrm{m}}=5.4$ marks a break redshift and $\dot{\rho}_{\mathrm{m}}=0.15 \mathrm{M}_{\odot} \mathrm{yr}^{-1} \mathrm{Mpc}^{-3}$ fixes the overall normalization. It is worth mentioning that these authors consider a $\Lambda \mathrm{CDM}$ model with the following parameters: $\Omega_{\mathrm{m}}=0.3, \Omega_{\Lambda}=0.7$, Hubble constant $H_{0}=100 h \mathrm{~km} \mathrm{~s}^{-1} \mathrm{Mpc}^{-1}$ with $h=0.7, \Omega_{\mathrm{b}}=0.04$ and a scaleinvariant primordial power spectrum with index $n=1$, normalized to the abundance of rich galaxy clusters at present day $\left(\sigma_{8}=0.9\right)$.

Hereafter, we denote the SFR density derived by such authors as S\&H SFR density. In Fig. 1, we plot the SFR as a function of the redshift, given by equation (14).

The S\&H SFR density is derived from hydrodynamic simulations of structure formation in $\Lambda \mathrm{CDM}$ cosmology. These authors study the history of cosmic star formation from the 'dark ages', at redshift $z \sim 20$ to the present. Besides gravity and ordinary hydrodynamics, they take into account radiative heating and cooling of gas, supernova feedbacks and galactic winds. The S\&H SFR thus derived is consistent with observations. It is important to bear in mind, however, that observations give information from at most a redshift around $z \sim 5$.

In the next section, we present the numerical results and discuss the detectability of the background of GWs. Looking at equation (5), one notes that, to integrate it, one still needs to set values for the following parameters: $\alpha$ and $\varepsilon_{\mathrm{GW}}$.

\section{DETECTABILITY OF THE BACKGROUND OF GRAVITATIONAL WAVES}

We have considered that the first stellar formation is related to the collapse of Population III objects. To evaluate the GW background produced by the formation of the black holes corresponding to the

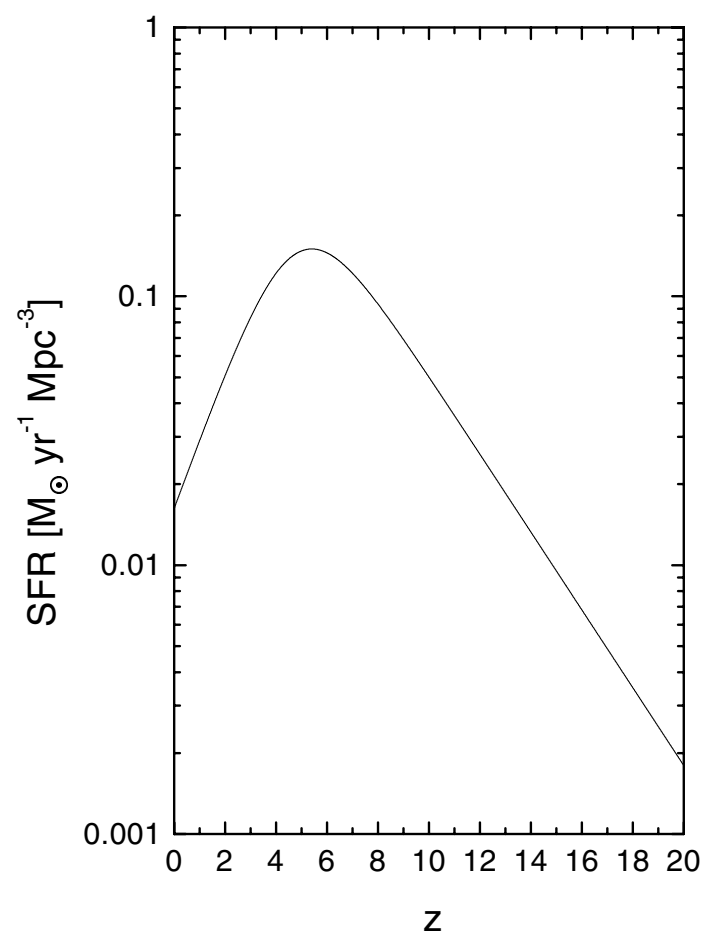

Figure 1. Springel \& Hernquist SFR density as a function of the redshift.

first stars, it is necessary to know the redshifts at which they began and finished being formed. This is a very hard question to answer, since it involves knowledge of the role of the negative and positive feedbacks of star formation, which are regulated by cooling and injection of energy processes. Here, as already discussed in preceding sections, we consider the history of star formation studied by Springel \& Hernquist (2003).

In particular, Springel \& Hernquist predicts a total stellar density at $z=0$ of $\Omega_{\star}=0.004$, corresponding to about 10 per cent of all baryons being locked up in long-lived stars, in agreement with recent determinations of the luminosity density of the Universe. In

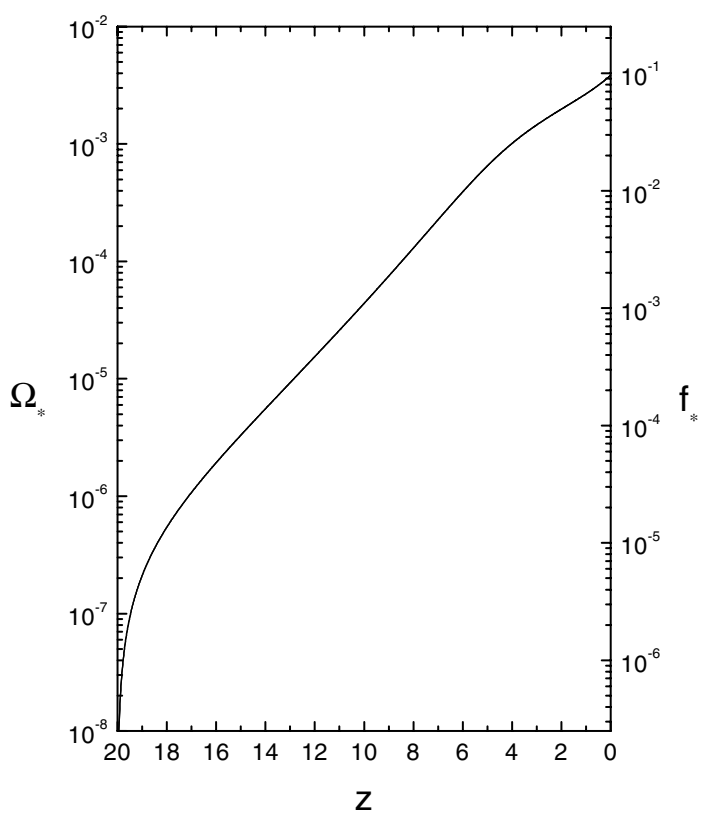

Figure 2. $\Omega_{\star}$ and $f_{\star}$ versus $z$ for the Springel \& Hernquist SFR density. 
Table 1. The GW frequency bands for different values of $\alpha$.

\begin{tabular}{lc}
\hline$\alpha$ & $\Delta v(\mathrm{~Hz})$ \\
\hline 0.1 & $50-5200$ \\
0.2 & $25-2600$ \\
0.3 & $16-1700$ \\
0.4 & $12-1300$ \\
0.5 & $9.9-1000$ \\
0.6 & $8.2-860$ \\
0.7 & $7.1-740$ \\
0.8 & $6.2-650$ \\
0.9 & $5.5-580$ \\
1.0 & $5.0-520$ \\
\hline
\end{tabular}

Fig. 2, we show $\Omega_{\star}$, and $f_{\star}\left(=\Omega_{\star} / \Omega_{\mathrm{B}}\right.$; the fraction of baryons in stars), as a function of the redshift for the S\&H SFR density.

Note that a relevant parameter in the equation for $h_{\mathrm{BG}}$, equation (5), is $\varepsilon_{\mathrm{GW}}$, the efficiency of production of GWs, whose distribution function is unknown. Thus, we have parametrized our results in terms of its maximum value, namely $\varepsilon_{\mathrm{GW}_{\max }}=7 \times 10^{-4}$, which is obtained from studies by Stark \& Piran (1986), who simulated the axisymmetric collapse of a rotating star into a black hole. We will see below that, if $\varepsilon_{\mathrm{GW}}$ is a very tiny fraction of the maximum value, the detection of the GW background whose existence we propose is very improbable, even for advanced antennas.

To calculate $h_{\mathrm{BG}}$, we still need to set a value for $\alpha$. It is worth noting that $\alpha$ may depend sensitively on the metallicity: the lower the value of $Z$ is, the higher the remnant masses are and the less ejected material there is relative to $Z_{\odot}$ stars. More realistically, there would be a dependence of $\alpha$ on the progenitor mass.

To assess the role of $\alpha$ in our results, we take into account different values for it. In Table 1 we present the GW frequency bands, $\Delta v$, for different values of $\alpha$. Note that $\Delta v$ is very sensitive to the value of $\alpha$.

Note that the frequency band is directly related to the remnant mass, the black hole mass, through equation (3). Then, once the GW background is observationally obtained, namely, $h_{\mathrm{BG}}$ versus $v_{\mathrm{obs}}$, it is possible to obtain the GW frequency band and, consequently, the black hole mass range.

In Fig. 3, an example is given on how the background of GWs, the strain per root Hertz, $\sqrt{S_{\mathrm{h}}}$, as a function of GW frequency $v$, depends on $\alpha$ and IMF.

Our results show that the frequency corresponding to the maximum value of $h_{\mathrm{BG}}, v_{\max }$, depends on $\alpha$. The larger $\alpha$ is, the lower $v_{\max }$ is; in fact, the latter is inversely proportional to the former.

Different values of $\alpha$ yield, besides shifting the GW frequencies to lower values, a background with larger amplitudes; in fact, note that $h_{\mathrm{BG}} \propto \alpha^{3 / 2}$, and also that $\sqrt{S_{\mathrm{h}}} \propto \alpha^{2}$.

To assess the role of the IMFs in our results, we consider different values of $x$, the Salpeter exponent. Different IMFs yield larger (lower) GW amplitudes if $x<1.35(x>1.35)$. Recall that, for $x<$ $1.35(x>1.35)$, the mass fraction of black holes is larger (lower) as compared with the standard IMF.

An important question is whether the background we study here is continuous or not. The duty cycle indicates if the collective effect of the bursts of GWs generated during the collapse of a progenitor star generates a continuous background. The duty cycle is defined as follows:

$\mathrm{DC}=\int_{z_{\mathrm{cf}}}^{z_{\mathrm{ci}}} \mathrm{d} R_{\mathrm{BH}} \overline{\Delta \tau_{\mathrm{GW}}}(1+z)$,

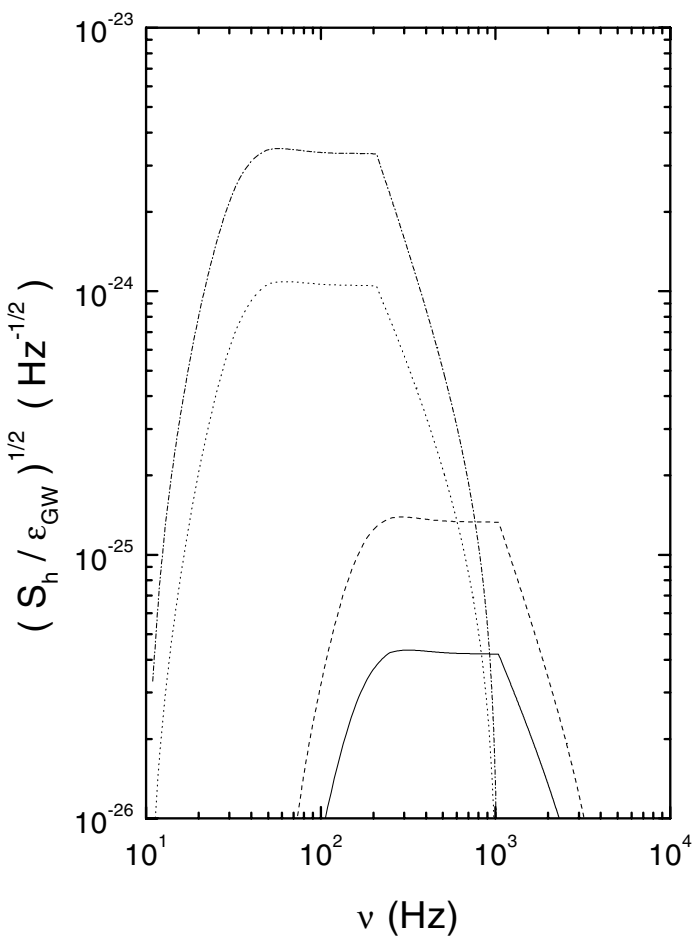

Figure 3. GW background spectra, $\sqrt{S_{\mathrm{h}}}$, as a function of GW frequency $\nu$, for different values of $\alpha$ and for different IMFs. The curves stand for the following models: standard IMF and $\alpha=0.1$ (solid line), $x=0.30$ and $\alpha=$ 0.1 (dashed line), standard IMF and $\alpha=0.5$ (dotted line), and $x=0.30$ and $\alpha=0.5$ (dash-dotted line).

where $\overline{\Delta \tau_{\mathrm{GW}}}$ is the average time duration of single bursts at the emission, which is inversely proportional to the frequency of the lowest quasi-normal mode of the rotating black holes (see, for example, Ferrari et al. 1999), which amounts to $\sim 1 \mathrm{~ms}$ for the mass range of the black holes considered here.

Because the star formation rate could be high, a significant amount of GWs could be produced. We also note that, independently of the primordial cloud mass and of the redshift of collapse, star formation occurring at high redshift could produce high duty cycle values, which lead us to conclude that the stochastic GW background could be continuous. For almost all the models studied here, the duty cycle is $\gg 1$.

It is worth noting, however, that the background predicted in the present study cannot be detected by single forthcoming interferometric detectors, such as VIRGO and LIGO (even by advanced ones). However, it is possible to correlate the signal of two or more detectors to detect the background that we propose exists. Michelson (1987) was the first to show that this kind of signal can, in principle, be detected by correlating the outputs of two different detectors. However, the main requirement that must be fulfilled is that they must have independent noise. This study was improved by Christensen (1992) and by Flanagan (1993). The reader should also refer to the papers by Allen (1997) and Allen \& Romano (1999), who also deal in detail with such an issue.

To assess the detectability of a GW signal, one must evaluate the signal-to-noise ratio $(\mathrm{S} / \mathrm{N})$, which for a pair of interferometers is given by (see, for example, Flanagan 1993; Allen 1997)

$(\mathrm{S} / \mathrm{N})^{2}=\left[\left(\frac{9 H_{0}^{4}}{50 \pi^{4}}\right) T \int_{0}^{\infty} \mathrm{d} v \frac{\gamma^{2}(v) \Omega_{\mathrm{GW}}^{2}(v)}{v^{6} S_{\mathrm{h}}^{(1)}(v) S_{\mathrm{h}}^{(2)}(v)}\right]$, 
Table 2. For different values of $\alpha$ we present the $\mathrm{S} / \mathrm{N}$ for pairs of LIGO I, II and III ('first', 'enhanced' and 'advanced', respectively) observatories for one year of observation. Note that an efficiency of generation $\varepsilon_{\mathrm{GW}_{\max }}=7 \times 10^{-4}$ is assumed.

\begin{tabular}{lccc}
\hline$\alpha$ & \multicolumn{3}{c}{ S/N } \\
& LIGO I & LIGO II & LIGO III \\
\hline 0.1 & $1.0 \times 10^{-6}$ & $4.1 \times 10^{-5}$ & $8.2 \times 10^{-5}$ \\
0.2 & $3.4 \times 10^{-5}$ & $2.6 \times 10^{-3}$ & $8.3 \times 10^{-3}$ \\
0.3 & $1.8 \times 10^{-4}$ & $1.9 \times 10^{-2}$ & $7.4 \times 10^{-2}$ \\
0.4 & $5.6 \times 10^{-4}$ & $6.2 \times 10^{-2}$ & 0.28 \\
0.5 & $1.3 \times 10^{-3}$ & 0.15 & 0.79 \\
0.6 & $2.6 \times 10^{-3}$ & 0.31 & 1.7 \\
0.7 & $4.4 \times 10^{-3}$ & 0.56 & 3.3 \\
0.8 & $6.3 \times 10^{-3}$ & 0.93 & 5.7 \\
0.9 & $8.3 \times 10^{-3}$ & 1.4 & 9.0 \\
1.0 & $1.1 \times 10^{-2}$ & 2.1 & 14 \\
\hline
\end{tabular}

where $S_{\mathrm{h}}^{(i)}$ is the spectral noise density, $T$ is the integration time, $\Omega_{\mathrm{GW}}$ is the closure energy density of the GW background (see, for example, de Araujo et al. 2002b) and $\gamma(v)$ is the overlap reduction function, which depends on the relative positions and orientations of the two interferometers. For the $\gamma(v)$ function, we refer the reader to Flanagan (1993), who was the first to calculate a closed form for the LIGO observatories. Flanagan (1993, see also Allen 1997) showed that the best window for detecting a signal is $0<v<64 \mathrm{~Hz}$, where the overlap reduction function has the greatest magnitude.

Here we consider, in particular, the LIGO interferometers. Their spectral noise densities have been taken from a paper by Owen et al. (1998) who in turn obtained them from Thorne, by means of private communication.

In Table 2, we present the $\mathrm{S} / \mathrm{N}$ for one year of observation for $\varepsilon_{\mathrm{GW}_{\max }}=7 \times 10^{-4}$ and different values of $\alpha$, for the three LIGO interferometer configurations.

Note that for the 'initial' LIGO (LIGO I), there is no hope of detecting the GW background we propose here, even for ideal orientation and locations of the interferometers, i.e. $|\gamma(v)|=1$. For the 'enhanced' LIGO (LIGO II), there is some possibility of detecting the background, since $\mathrm{S} / \mathrm{N}>1$, if $\varepsilon_{\mathrm{GW}}$ is around the maximum value and $\alpha>0.8$. Even if the LIGO II interferometers cannot detect such a background, it will be possible to constrain the efficiency of GW production and $\alpha$.

The prospect for the detection with the 'advanced' LIGO (LIGO III) interferometers is much more optimistic, because the $\mathrm{S} / \mathrm{N}$ for $\alpha>0.5$ is significantly greater than unity. Only if the value of $\varepsilon_{\mathrm{GW}}$ were significantly lower than the maximum value would the detection not be possible. In fact, the $\mathrm{S} / \mathrm{N}$ is critically dependent on this parameter, whose distribution function is unknown.

Our results thus show that the $\mathrm{S} / \mathrm{N}$ is sensitive to variations of $\alpha$. The larger $\alpha$ is, the lower the GW frequencies are and the higher $h_{\mathrm{BG}}$ is, and because the best window for detection is around $0<$ $v<64 \mathrm{~Hz}$, the higher $\mathrm{S} / \mathrm{N}$ is.

Even if $\alpha$ is not known beforehand, it is possible to impose a constraint on its values. For example, if one found from GW observations that the GW frequency band was $10-1000 \mathrm{~Hz}$, one would obtain (using equation 3 ) that $\alpha \simeq 0.5$.

It would be interesting to perform a study considering $\alpha$ as a function of the progenitor mass, which would result in a more realistic model. There are some studies in the literature considering how the remnant mass depends on the progenitor (see, for example, Fryer \&
Kalogera 2001), but we leave this issue for another study to appear elsewhere. Thus, the values of $\alpha$ presented in Table 1 should be considered mean values of the fraction of mass which forms black holes from progenitor stars ranging from $m_{\min }-m_{\mathrm{u}}$.

Note that the $\mathrm{S} / \mathrm{N}$ for a given SFR density, IMF and $\alpha$, and for one year of observation, still presents a dependence on $\varepsilon_{\mathrm{GW}}$, namely

$\mathrm{S} / \mathrm{N} \propto \varepsilon_{\mathrm{GW}}$.

As already mentioned, the $\varepsilon_{\mathrm{GW}}$ distribution function is unknown. We have adopted here the maximum value as a reference, but if its actual value is much less than this value, the $\mathrm{S} / \mathrm{N}$ could be lower than unity for all the models studied here, even for a LIGO III pair. Let us think of what occurs with other compact objects - namely, the neutron stars - to see if we can learn something from them. Hot and rapidly rotating neutron stars can lose angular momentum to gravitational radiation via the so-called r-mode instability (Anderson 1998). This could explain why all known young neutron stars are relatively slow rotators. The black holes could have had a similar history, i.e. they could have been formed rapidly rotating and lost angular momentum to gravitation radiation via their quasi-normal modes. If this was the case, the value of $\varepsilon_{\mathrm{GW}}$ could be near the maximum one, or in the worst case, it could have a value to produce $\mathrm{S} / \mathrm{N}>1$ at least for a LIGO III pair.

In order to assess the values of $\alpha$ and $\varepsilon_{\mathrm{GW}}$ that yield $\mathrm{S} / \mathrm{N}>1$, for a given SFR density and IMF, we present in Fig. 4 the regions in the $\left(\alpha, \varepsilon_{\mathrm{GW}}\right)$ plane where $\mathrm{S} / \mathrm{N}$ could be greater than unity for a pair of LIGO III interferometers.

Note that we present two curves, one for the standard Salpeter IMF, namely, with $x=1.35$, and another one for $x=0.30$. This latter value for $x$ yields 10 times the mass fraction of black holes of the standard IMF.

Note that unless $\varepsilon_{\mathrm{GW}}$ is very small, $\mathrm{S} / \mathrm{N}$ can be significantly greater than unity, for reliable values of $\alpha$, namely $\alpha>0.5(\alpha>0.3)$ for

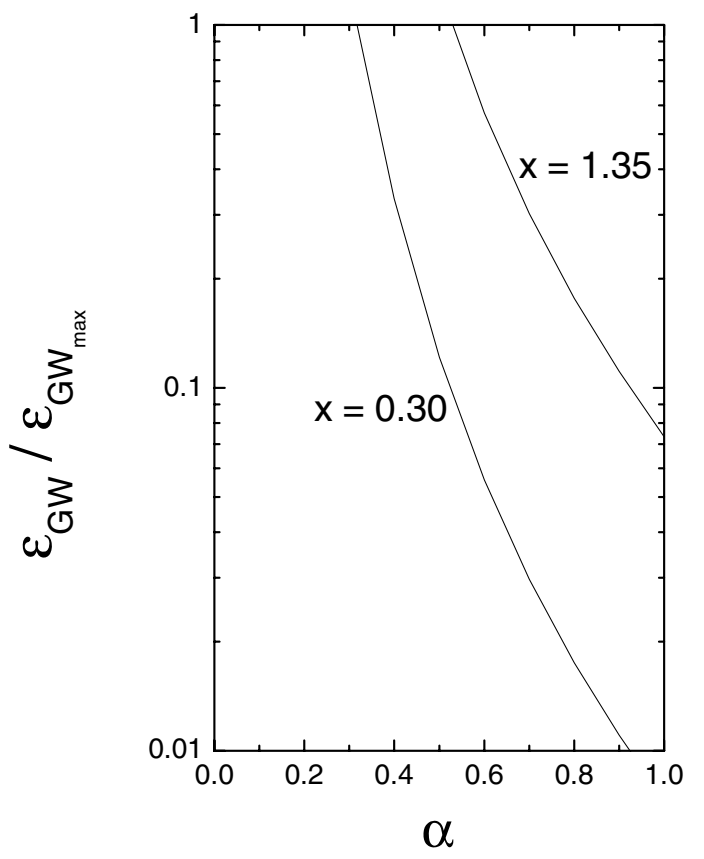

Figure 4. Relative efficiency of $\mathrm{GW}$ generation, $\varepsilon_{\mathrm{GW} / \varepsilon_{\mathrm{GW}}}$, as a function of the fraction of the progenitor mass which forms the remnant black hole, $\alpha$, for two different IMFs, namely, $x=1.35$ and $x=0.30$. The curves represent where $\mathrm{S} / \mathrm{N}=1$; above them, $\mathrm{S} / \mathrm{N}>1$. We assume $\varepsilon_{\mathrm{GW}_{\max }}=7 \times 10^{-4}$. 
$x=1.35(x=0.30)$, indicating that the background could, in principle, be detected in the near future.

\section{CONCLUSIONS}

We present here a study concerning the generation of GWs produced from a population of black holes in the Springel \& Hernquist scenario for the history of cosmic star formation. Our results show that the formation of a population of black holes and a significant stochastic GW background associated with them is generated.

We consider that stars are formed following a Salpeter IMF and having masses in the range $0.1-125 \mathrm{M} \odot$. Certainly, the results presented here are dependent on this particular choice. A steeper IMF would modify the number of high-mass stars, modifying $h_{\mathrm{BG}}$ and the frequency band of the GWs (see, for example, Fig. 3).

As seen, with reasonable parameters, our results show that a significant amount of GWs is produced related to the history of cosmic star formation studied by Springel \& Hernquist, and can, in principle, be detected by a pair of LIGO II (or most probably by a pair of LIGO III) interferometers. However, a relevant question should be considered: what astrophysical information can one obtain, whether (or not) such a putative background is detected?

First, let us consider a non-detection of the GW background. The critical parameter to be constrained here is $\varepsilon_{\mathrm{GW}}$. A non-detection would mean that the efficiency of GWs during the formation of black holes is not high enough.

Secondly, a detection of the background with a significant $\mathrm{S} / \mathrm{N}$ would permit us to obtain the curve $h_{\mathrm{BG}}$ versus $v_{\mathrm{obs}}$. As the $\alpha$ parameter is sensitive to the GW frequency band $(\Delta v)$ and to the position of the $h_{\mathrm{BG}}$ peak $\left(h_{\mathrm{BG}} \propto \alpha^{3 / 2}\right)$, we could impose constraints on the value of $\alpha$.

For a given value of $\alpha$ and considering an IMF compatible with the scenario proposed, for example by $\mathrm{S} \& \mathrm{H}$, one can also constrain the value of $\varepsilon_{\mathrm{GW}}$.

In particular, we have tried to show in this paper that from a specific SFR density model, there are several other parameters related to the formation of the first stars that could be obtained or constrained.

\section{ACKNOWLEDGMENTS}

ODM and ODA would like to thank the Brazilian agency FAPESP for support (grants 02/07310-0 and 02/01528-4; 98/13468-9, respec- tively). JCNA and ODA thank the Brazilian agency CNPq (Brazil) for partial financial support (grants 304666/02-5, 300619/92-8, respectively). We thank the referee for helpful comments, and for calling our attention to the fact that because the SFR is calculated in the rest frame at redshift $z$, it must be redshifted in order to properly account for the resulting gravitational waves at redshift zero.

\section{REFERENCES}

Allen B., 1997, in Lasota J.-P., Mark J.-A., eds, Relativistic Gravitation and Gravitational Radiation. Cambridge Univ. Press, Cambridge, p. 373

Allen B., Romano J. D., 1999, Phys. Rev. D, 59, 102001

Anderson N., 1998, ApJ, 211, 708

Bromm V., Coppi P. S., Larson R. B., 1999, ApJ, 527, L5

Bromm V., Coppi P. S., Larson R. B., 2002, ApJ, 564, 23

Christensen N., 1992, Phys. Rev. D, 46, 5250

de Freitas-Pacheco J. A., 1998, AJ, 116, 1701

de Araujo J. C. N., Miranda O. D., Aguiar O. D., 2000, Phys. Rev. D, 61, 124015

de Araujo J. C. N., Miranda O. D., Aguiar O. D., 2001, ApJ, 350, 368

de Araujo J. C. N., Miranda O. D., Aguiar O. D., 2002a, Classical and Quantum Gravity, 19, 1335

de Araujo J. C. N., Miranda O. D., Aguiar O. D., 2002b, MNRAS, 330, 651

Ferrari V., Matarrese S., Schneider R., 1999, MNRAS, 303, 247

Flanagan E. E., 1993, Phys. Rev. D, 48, 2389

Fryer C. L., Kalogera V., 2001, ApJ, 554, 548

Gilmore G., 2001, in Tacconi L., Lutz D., eds, Starburst Galaxies: Near and Far. Springer-Verlag, Heidelberg, p. 34 (astro-ph/0102189)

Mackey J., Bromm V., Hernquist L., 2003, ApJ, 586, 1

Michelson P. F., 1987, MNRAS, 227, 933

Owen B. J., Lindblom L., Cutler C., Schutz B. F., Vecchio A., Andersson N., 1998, Phys. Rev. D, 58, 084020

Scalo J., 1998, in Gilmore G., Howell D., eds, ASP Conf. Ser. Vol. 142, The Stellar IMF. Astron. Soc. Pac., San Francisco, p. 201

Springel V., Hernquist L., 2003, MNRAS, 339, 312

Stark R. F., Piran T., 1986, in Ruffini R., ed., Proc. Fourth Marcel Grossmann Meeting on General Relativity. Elsevier Science, Amsterdam, p. 327

Thorne K. S., 1987, in Hawking S. W., Israel W., eds, 300 Years of Gravitation. Cambridge Univ. Press, Cambridge, p. 331

Venkatesan A., 2000, ApJ, 537, 55

This paper has been typeset from a $\mathrm{T}_{\mathrm{E}} \mathrm{X} / \mathrm{LT} \mathrm{E} \mathrm{X}$ file prepared by the author. 